\title{
Meteoroid impacts onto asteroids: a competitor for Yarkovsky and YORP
}

\author{
Paul A. Wiegert \\ Dept. of Physics and Astronomy, The University of Western Ontario, London Ontario \\ CANADA \\ Submitted to Icarus July 142014
}

\begin{abstract}
The impact of a meteoroid onto an asteroid transfers linear and angular momentum to the larger body, which may affect its orbit and its rotational state. Here we show that the meteoroid environment of our Solar System can have an effect on small asteroids that is comparable to the Yarkovsky and YarkovskyO'Keefe-Radzievskii-Paddack (YORP) effects under certain conditions.

The momentum content of the meteoroids themselves is expected to generate an effect much smaller than that of the Yarkovsky effect. However, momentum transport by ejecta may increase the net effective force by two orders of magnitude for impacts into bare rock surfaces. This result is sensitive to the extrapolation of laboratory microcratering experiment results to real meteoroid-asteroid collisions and needs further study. If this extrapolation holds, then meteoroid impacts are more important to the dynamics of small asteroids than had previously been considered.
\end{abstract}

Asteroids orbiting on prograde orbits near the Earth encounter an anisotropic meteoroid environment, including a population of particles on retrograde orbits generally accepted to be material from long-period comets spiralling inwards under Poynting-Robertson drag. High relative speed $(60 \mathrm{~km} / \mathrm{s})$ impacts by meteoroids provide a small effective drag force that decreases asteroid semimajor axes and which is independent of their rotation pole. This effect may exceed the instantaneous Yarkovsky drift at sizes near and below one meter.

The rate of reorientation of asteroid spins is also substantially increased 
when momentum transport by ejecta is included. This has an indirect effect on the net Yarkovsky drift, particularly the diurnal variant, as the sign of the drift it creates depends on its rotational state. The net drift of an asteroid towards a resonance under the diurnal Yarkovsky effect can be slowed by more frequent pole reorientations. This may make the effect of the meteoroid environment more important than the Yarkovsky effect at sizes even above one meter.

Meteoroid impacts also affect asteroid spins at a level comparable to that of YORP at sizes smaller than tens of meters. Here the effect comes primarily from a small number of impacts by centimeter size particles. We conclude that recent measurements of the YORP effect have probably not been compromised, because the targets large sizes and because they are known or likely to be regolith-covered rather than bare rock. However, the effect of impacts increases sharply with decreasing size, and will likely become important for asteroids smaller than a few tens of meters in radius.

Keywords: near-Earth objects; asteroid Itokawa; asteroid 2000 PH5; asteroid dynamics; asteroids rotation; celestial mechanics; impact processes, meteorites; meteors

\section{Introduction}

The study of the delivery of meteorites to Earth was much advanced by the revival of the notion that the uneven re-radiation of incident sunlight could affect the orbits of small asteroids. Known as the Yarkovsky effect, this phenomenon results when temperature differences on an asteroid's surface result in it reradiating energy (and hence momentum) asymmetrically. The Yarkovsky effect has been widely discussed elsewhere (the reader is directed to Rubincam (1998) and Farinella et al. (1998) for excellent reviews). It is of interest here because it is one of few dynamical effects acting in the main asteroid belt which create a net trend in the semimajor axis $a$ of an asteroid's orbit. If such a change in $a$ moves the body into a mean-motion or other resonance, its orbit may be dramatically changed as a result. Resonances can eject asteroids from the asteroid belt and 
play a key role in the delivery of meteorites to Earth. Thus the Yarkovsky effect, while itself creating only a small change in asteroid orbits, is nonetheless crucial in moving meteorite parent bodies from the asteroid belt to near-Earth space. The importance of the Yarkovsky effect leads one to consider whether or not other small effects might have important roles in the evolution of small asteroids. Here we consider the effect of momentum transfer via meteoroid impacts on small asteroids and show that it can compete with the Yarkovsky effect (and its cousin, the Yarkovsky-O'Keefe-Radzievskii-Paddack or YORP effect) under certain conditions.

In section 2 we will introduce the meteoroid environment near the Earth. In section 3 the dynamical effects of meteoroid impacts on small asteroids, and in particular the role of momentum transport by ejecta, will be discussed and comparisons drawn with the Yarkovsky effect. Section 4 extends the discussion to the YORP effect, section 5 considers radiation pressure and rates of erosion and conclusions are drawn in section 6

\section{Meteoroid environment at Earth}

Most of the mass accreted by the Earth is in smaller particles, at least over short times. Larger individual asteroid impacts may dominate the overall mass input to the Earth (Rabinowitz, 1993; Rabinowitz et al., 1993) on million year timescales but they are not relevant here. Love \& Brownlee (1993) determined that meteoroids with mass $m \approx 1.5 \times 10^{-8} \mathrm{~kg}$ corresponding to a radius $r=$ $220 \mu \mathrm{m}$ at a density $\rho_{p}=2500 \mathrm{~kg} \mathrm{~m}^{-3}$ dominate the meteoroid flux at Earth. Earlier studies such as those of Grün et al. (1985) found similar values though with total fluxes somewhat (2-3 times) lower.

At these sizes, the meteoroid environment of the Earth is asymmetric. This is partly because of the Earth's motion around the Sun: our planet tends to get hit more on the leading side than the trailing side. However the asymmetry also originates in part from a heterogeneous distribution of particle orbits. Studies of the sporadic meteors (that is, those meteors distinct from meteor showers) 
show concentrations of meteoroid orbits towards the direction of the Earth's motion around the Sun (e.g. Stohl (1986); Brown \& Jones (1995); Chau et al. (2007); Campbell-Brown (2008) and many others). When displayed in a comoving reference frame centred on the apex of the Earth's way, a number of concentrations of impinging orbits are discerned. Here we will be most interested in those known as the north and south apex sporadic meteor sources.

Meteoroids arriving at Earth from these apex sources have relative velocities peaking at $60 \mathrm{~km} / \mathrm{s}$ (Jones \& Brown, 1993; Chau et al., 2007). These particles are on approximately circular retrograde orbits. Attributed to long-period and Halley-family cometary debris that has decayed onto low-eccentricity orbits through Poynting-Robertson drag, these particles constitute the dominant momentum and kinetic energy flux in near-Earth space. Because they arrive from the direction of the Earth's motion, they hit our planet essentially head-on and provide a small but consistent tangential drag force on any body (such as an asteroid) on a similar orbit. Though the meteoroid environment at the asteroid belt is not well known, it is reasonable to assume that it is similar to that at Earth and will also produce a net drag on asteroidal bodies.

The fraction of retrograde meteoroids arriving at Earth has been measured but there are still uncertainties. Radial scatter meteor radars (often called "High Power Large Aperture" or HPLA radars) typically see a larger fraction of apex meteors (> 80\%) (e.g. Sato et al., 2000; Hunt et al., 2004; Janches et al., 2003; Chau \& Woodman, 2004) while transverse scatter (or "meteor patrol") radars, typically see a smaller fraction $(\sim 50 \%)$ (e.g. Taylor, 1995; Galligan \& Baggaley, 2004) as do video meteor systems (Campbell-Brown \& Braid, 2011). This effect can be attributed to the different instrumental sensitivities (Wiegert et al., 2009) at different particle sizes and speeds; however here for simplicity we will assume that the apex meteoroids constitute a fraction $s=50 \%$ of the meteoroid population at these sizes. The magnitude of the effect of this idealized meteoroid environment on a target asteroid will be calculated first at Earth.

If the meteoroid flux at the Earth is dominated by the apex source as studies of the sporadic meteors would suggest, then taking the (cumulative) flux from 
Fig. 3 of Love \& Brownlee (1993), where their differential flux peaks $(m \approx$ $1.5 \times 10^{-8} \mathrm{~kg}$ ) we get $n \approx 3 \times 10^{-8} \mathrm{~m}^{-2} \mathrm{~s}^{-1}$ where $n$ is the flux of particles per square meter per second, and $m$ is the particle mass. Given these conditions, a one-meter radius asteroid on a circular orbit near the Earth sees roughly three impacts per year, and each of impactor carries $\sim 10^{-12}$ of the momentum of the target. We will consider their cumulative effect to be a small effective drag on the target asteroid.

\section{Effective drag due to meteoroid impacts}

The impact of a small meteoroid onto an asteroid surface transfers kinetic energy and momentum to the larger body. Using the impulse approximation, the force $F$ exerted on the asteroid as a result of a momentum gain $\Delta p$ during a time $\Delta t$ is $F=\Delta p / \Delta t$. The fraction $\eta$ of the incoming momentum received by the target is unity in the case of a completely inelastic collision, and could be as high as two in the case of an elastic collision. However, high-velocity impacts are highly inelastic and we will adopt $\eta \approx 1$.

The acceleration $f_{a}=F / M$ imparted to an assumed spherical asteroid of mass $M$, density $\rho_{a}$ and radius $R$ being impacted head-on by the apex meteoroid population as described earlier would be

$$
f_{a}=\frac{s n m v \pi R^{2}}{\frac{4}{3} \pi \rho_{a} R^{3}}=\frac{3 s n m v}{4 R \rho_{a}}
$$

where $v$ is the relative velocity.

Lagrange's planetary equations e.g. Roy (1978) can be used to calculate the resulting change in semimajor axis $a$ for an asteroid with zero eccentricity and inclination that is subject to a tangential acceleration such as that of Eq. 1

$$
\dot{a} \approx-\frac{2 f_{a}}{n^{\prime}}=-\frac{3 s n m v a^{3 / 2}}{2 \sqrt{G M_{\odot}} R \rho_{a}}
$$

where $n^{\prime}=\sqrt{G M_{\odot} / a^{3}}$ is the asteroid's mean motion. For an $R=1 \mathrm{~m}$ target asteroid at $1 \mathrm{AU}$, the apex meteoroid environment produces a decrease in 
semimajor axis of

$$
\dot{a} \approx-6.1 \times 10^{-6}\left(\frac{s}{0.5}\right)\left(\frac{R}{1 \mathrm{~m}}\right)^{-1}\left(\frac{\rho_{a}}{3500 \mathrm{~kg} \mathrm{~m}^{-3}}\right)^{-1} \mathrm{AU} \mathrm{Myr}^{-1}
$$

This effective drag force is much lower than that of the Yarkovsky effect in its different variants, by factors of several up to 100 (e.g. Figure 1 of Farinella et al., 1998).

Though direct momentum transfer as described above may be negligible compared to the Yarkovsky effect, there are two subsidiary effects that may make small asteroids' interactions with the meteoroid environment important. First, we will show that the ejecta produced by the impact results in a much larger momentum transfer to the target than simply that carried by the impactor, magnifying the effective force. Secondly, this may also shorten the timescale between collisional re-orientation of the asteroid's spin axis, an important consideration for the Yarkovsky effect, particularly the diurnal variant.

\subsection{Momentum transport by ejecta}

A hypervelocity impact creates a crater on the target, and the amount of mass removed during this process is often larger than the mass of the projectile itself. The incoming particle is vapourized on impact since its kinetic energy content vastly exceeds its internal binding energy, and the resulting explosive event excavates a crater in the target. Consider the impact as seen in the reference frame of the centre of mass of the impactor-target pair. Taking the impactor's mass to be $m$ and its impact velocity $v$, a fraction $\epsilon$ of the impactor's kinetic is converted to kinetic energy of motion of the ejecta and target, resulting the ejection of a mass $N m$ of target material at a velocity $\gamma v$, where $N$ and $\gamma$ are multiplicative factors that depend on the detailed physics of impact.

After the impact, the ejecta carries away momentum $\gamma N m v$ which by Newton's Third Law is balanced by an opposite momentum transfer to the target. The ratio of the momentum of the ejecta to that of the projectile itself we call $\alpha=N \gamma$. If $\alpha>1$, then the mobilization of ejecta creates an effective force that exceeds that due simply to the momentum content of the projectile. Note 
that $\alpha>1$ does not violate conservation of momentum. The kinetic energy of the impactor provides energy for the release of ejecta, and it is conservation of momentum between the ejecta and the target that provide the drag force that we consider here.

The value of $\alpha$ can be related to $\epsilon, N$ and $\gamma$. Our definition of $\epsilon$ implies that

$$
\frac{1}{2} \epsilon m v^{2}=\frac{1}{2} N m(\gamma v)^{2}+\frac{1}{2} M V^{2}
$$

where $M$ is the mass of the target after impact, and $V$ its speed. The ratio of the kinetic energy acquired by the target to that of the ejecta is

$$
\frac{\frac{1}{2} M V^{2}}{\frac{1}{2} N m(\gamma v)^{2}}=\frac{M V^{2}}{N m(\gamma v)^{2}}
$$

Conservation of momentum implies that

$$
\begin{aligned}
(N m)(\gamma v) & =M V \\
V & =\frac{\gamma N m v}{M}
\end{aligned}
$$

which when substituted back into Eq. 5 gives

$$
\begin{aligned}
\frac{M V^{2}}{N m(\gamma v)^{2}} & =\frac{M\left(\frac{\gamma N m v}{M}\right)^{2}}{N m(\gamma v)^{2}} \\
& =\frac{N m}{M}
\end{aligned}
$$

The target carries only a fraction $N m / M$ of the kinetic energy which is negligible in the limit that the target mass is much larger than the amount of mass released by the impact. Since this applies to most of the impacts we consider here, this allows us to simplify Eq. 4 to

$$
\begin{aligned}
\frac{1}{2} \epsilon m v^{2} & \approx \frac{1}{2} N m(\gamma v)^{2} \\
\epsilon & \approx N \gamma^{2}
\end{aligned}
$$

From this, we obtain $\gamma \approx \sqrt{\epsilon / N}$ and

$$
\alpha=N \gamma \approx \sqrt{\epsilon N}
$$

We will see below that for typical microcratering events expected on asteroids, $N$ is very large $\left(\gtrsim 10^{4}\right)$ and $\alpha \sim 100$, which pushes the resulting drag force 
into a range comparable to that of the Yarkovsky effect for small asteroids. This is a linchpin argument of this paper, namely that momentum transport by ejecta creates a substantially larger effective force on the target than if the simple momentum content of the projectile would imply. Since hypervelocity cratering is complex, our analysis may be over-simplified and further study by experts in that field is greatly encouraged by this author.

\subsection{Microcratering experiments}

Microcratering experiments involve accelerating of particles to high speeds in the lab and directing them onto targets composed of the materials of interest. Such experiments often measure the amount of material excavated $(N)$, while the values for ejecta velocities $(\gamma)$ and energies $(\epsilon)$ are less well-studied. We will use experimental measures of $N$ and $\epsilon$, seeming to be the best constrained of the three, to estimate $\gamma$ and show that our value of $\gamma$ is consistent with those experiments that have measured ejecta velocities.

Here we consider the same target cases as Farinella et al. (1998) who provide a very clear exposition of the effects of the Yarkovsky effect on meter-class asteroids of various types. We assume our target asteroids are either bare rock, regolith-covered rock or bare iron. Bare or regolith-covered rock are the best studied in terms of microcratering experiments, having received much attention during the Apollo era e.g. Fechtig et al. (1972).

For the case of bare rock, perhaps the most likely situation for a meteorite parent body, Gault (1973) provides empirically-based formulae for the displaced mass as a function of the kinetic energy. After firing a variety of projectiles (densities of $0.95-7.8 \mathrm{~g} \mathrm{~cm}^{-3}$ ) into a selection of terrestrial rocks (including basalts) as well as the Indarch meteorite (range of target densities: $2.5-5 \mathrm{~g} \mathrm{~cm}^{-3}$ ) at high-velocity and normal incidence, the mass displaced $M_{e}$ in grams was found to be

$$
M_{e}=10^{-10.061}\left(\frac{\rho_{p}}{\rho_{a}}\right)^{1 / 2}(K E)^{1.133}
$$

where $\rho_{p}$ is the projectile density $\left(\mathrm{g} \mathrm{cm}^{-3}\right), \rho_{a}$ is the target density $\left(\mathrm{g} \mathrm{cm}^{-3}\right)$ and $K E$ is the kinetic energy in ergs. Gault's formula is applicable to craters with 
diameters from $10^{-3}$ to $10^{3} \mathrm{~cm}$ with impact kinetic energies of $10-10^{12}$ ergs. An impact by a $1.5 \times 10^{-8} \mathrm{~kg}$ particle at $60 \mathrm{~km} / \mathrm{s}$ has an energy of $2.7 \times 10^{8} \mathrm{ergs}$ and falls squarely in this range. The resulting $N=M_{e} / m$ is

$$
N \approx 2.1 \times 10^{4}\left(\frac{\rho_{p}}{\rho_{a}}\right)^{1 / 2}\left(\frac{m}{1.5 \times 10^{-8} \mathrm{~kg}}\right)^{0.133}\left(\frac{v}{6 \times 10^{4} \mathrm{~m} \mathrm{~s}^{-1}}\right)^{2.266}
$$

A hypervelocity impact can displace four orders of magnitude more mass than that of the projectile. For the case of dense minerals and glass, microcratering is often accompanied by large spalled regions, annuli around the crater itself where material fractures off in large plates or flakes (e.g. Hörz et al., 1971) and this contributes to the relatively large mass displaced.

The fraction of impactor kinetic energy that goes into the motion of the ejecta has been found experimentally to be small for low impact speeds but to increase sharply as speed increases. Braslau (1970) found $\epsilon \approx 0.5$ both for dry quartz sand and basalt at impact speeds of $6 \mathrm{~km} / \mathrm{s}$. Later studies by Hartmann (1983) were somewhat critical of Braslau's assumptions but still found $\epsilon \approx 0.3$ as the impact speed increased to $4 \mathrm{~km} / \mathrm{s}$. Here we will adopt $\epsilon=0.5$ since we are considering even higher speeds, while noting that our expression for $\alpha$ is relatively insensitive to its precise value, going only like $\epsilon^{1 / 2}$ (Eq. 12).

Together with Eq. 11] and 14 this allow us to estimate $\gamma \approx \sqrt{\epsilon / N} \approx 5 \times 10^{-3}$. This value, which implies ejecta velocities around $0.3 \mathrm{~km} \mathrm{~s}^{-1}$ for a $60 \mathrm{~km} \mathrm{~s}^{-1}$ impact, is consistent with experimental measurements of late-stage ejecta from loose sand targets (Braslau, 1970) and powdered targets of pumice and basalt (Hartmann, 1985), though the ejecta were found to have a wide range of velocities.

From the values for $\epsilon$ and $N$, we deduce an $\alpha \approx \sqrt{\epsilon N} \approx 10^{2}$ (from Eq. 12) for bare rock: the cratering process can release two orders of magnitude more momentum than is carried by the projectile itself. As a result, Eq. 3 should be multiplied by a factor $\alpha \sim 100$, which makes it competitive with the Yarkovsky effect under some conditions (this will be discussed in more detail in section 3.3). We again note that our analysis is based on an extrapolation of microcratering results beyond the impact speeds actually examined in the lab, and a more 
detailed examination of the phenomenon is certainly warranted.

Impacts into mineral dust, which would be more applicable to regolithcovered bodies, are similar though the displaced masses are often lower. Highvelocity impacts by centimeter sized particles displace two to four orders of magnitude more mass than that of the projectile (references in Vedder (1972) incl. Braslau (1970) ) with loosely-packed material being more easily displaced than packed or consolidated material. For 2 to 5 micron-sized polystyrene (1.06 g $\mathrm{cm}^{-3}$ ) spheres accelerated to 2.5 to $12 \mathrm{~km} / \mathrm{sec}$ into mineral dust, the amount of displaced mass was three orders of magnitude larger than the impactor mass, though the granular nature of the target resulted in large $( \pm 50 \%)$ errors in displaced mass. For impacts into granular material, we conclude that $N$ may be lower, reducing $\alpha$. Since small meteorite parent bodies are expected to be bare rock, we will adopt $\alpha=100$ here as our fiducial value for stony targets, recognizing that it may be smaller if the targets are actually covered with regolith.

For the bare iron case, Comerford (1967) found that $2.6 \mathrm{~km} / \mathrm{s}$ silicon carbide particles eroded the Hoba iron meteorite at least two orders of magnitude more slowly than the Indarch chondrite meteorite, implying $N \lesssim 200$ from simple extension of Eq. 14, Schaeffer et al. (1979) fired $1.5-2 \mathrm{~mm}$ steel and sapphire particles into the Gibeon iron meteorite and also found that plastic flow significantly reduced the amount of material released. They concluded that the erosion rate of iron meteorites was a factor of 10 slower than that of stony ones $(N \sim 2000)$.

Comerford (1967) points out that much depends here on the brittle versus ductile nature of the target, which is sensitive to crystal size and temperature. Higher velocity impacts at the lower temperatures of the asteroid belt may move into the brittle fracture (instead of the ductile flow) regime which may release more ejecta. However it seems unlikely that $N$ values approaching those of rock targets would be reached. If we adopt the values of $N \sim 200$ and take $\epsilon=0.5$ as before, we have $\alpha=10$ for iron meteorite parents, recognizing it contains a substantial uncertainty. Nonetheless, even on iron targets, it seems that significantly more momentum may be carried by the ejecta than is received 
from the impinging particle.

If ejecta do generally carry away 10-100 times as much momentum as is imparted by the meteoroid, then Eq. [3 is 10-100 times higher, and becomes competitive with the Yarkovsky effect. Before comparing them side-by-side, let us first extrapolate the near-Earth meteoroid environment to the asteroid belt.

\subsection{Extrapolation to the asteroid belt}

Equation 3 assumes a meteoroid environment like that at Earth. If we are interested in whether or not impact drag competes with the Yarkovsky effect in the delivery of small asteroids to resonance 'escape hatches' in the asteroid belt, we need to consider the meteoroid environment there. Unfortunately, there is little experimental data on the asteroid belt's meteoroid environment. Here we simply assume that the environment at Earth has evolved directly from that at the asteroid belt under PR drag.

The apex meteoroids encountered by our planet are on roughly circular retrograde orbits (Jones \& Brown, 1993; Chau et al., 2007), and thought to be particles released from retrograde comets whose semimajor axis $a$ and eccentricity $e$ have decayed through Poynting-Robertson drag. We assume here that the asteroid belt hosts the same meteoroids (at an earlier time) as they spiral inwards to produce the meteoroids observed at Earth, and that we can estimate the properties of the asteroid belt meteoroid environment from meteor observations taken here.

Returning to Eq. 2, let's assume that the impactor mass $m$, the target radius $R$ and density $\rho_{a}$ are not functions of heliocentric distance. The factors that are include the velocity of each impact $v$, which goes like $a^{-1 / 2}$ owing to Kepler's Third Law. The flux of impactors $n$ is also affected: it is proportional to $v$ times the number density of particles $n_{p}$. Assuming the meteoroids are spiralling inwards under PR drag near the ecliptic plane, the vertical and azimuthal densities of particles both go like $a^{-1}$ while the radial density is proportional to $1 / \dot{a}_{P R}$, where $\dot{a}_{P R}$ is the rate at which the meteoroids spiral inwards under PR drag. The effect of PR drag on particle semimajor axis is given by 
Weidenschilling \& Jackson (1993) to be $\dot{a}_{P R} \propto a^{-1}$ for circular orbits. This result of these factors is that $n_{p} \propto a^{-1}$, and the flux $n=v n_{p} \propto a^{-3 / 2}$. Eq. 2 would increase like $a^{3 / 2}$ if $n$ and $v$ were constant, but $\dot{a}$ will instead decrease slightly (as $a^{-1 / 2}$ ) with increasing semimajor axis.

When extended to the distance of the main asteroid belt, Equation 3 becomes $\dot{a}=-4.3 \times 10^{-4}\left(\frac{s}{0.5}\right)\left(\frac{\alpha}{100}\right)\left(\frac{a}{2 \mathrm{AU}}\right)^{-1 / 2}\left(\frac{R}{1 \mathrm{~m}}\right)^{-1}\left(\frac{\rho_{a}}{3500 \mathrm{~kg} \mathrm{~m}^{-3}}\right)^{-1} \mathrm{AU} \mathrm{Myr}^{-1}$

Eq. 15 is expressed at the same heliocentric semimajor axis (2 AU) considered by Farinella et al. (1998) in their analysis of the Yarkovsky effect, which allows us to compare our results directly with theirs.

For bare rock (Fig. 1) impact drag exceeds two of the three variants of the Yarkovsky effect considered by Farinella et al. (1998) (the seasonal effect and the diurnal effect under the assumption of size-dependent spin) but only at sizes of tens of centimeters. The drag effect considered here is smaller than the diurnal effect in the case where the spins of small asteroids are independent of their sizes, though Farinella et al. (1998) indicate that size dependent spin states are more realistic in their opinion. If Farinella et al. (1998) are right and spin rates are typically higher for smaller asteroids, then the effect presented here is of the same order of magnitude as the Yarkovsky effect for meter class asteroids, while the seasonal effect dominates at larger sizes.

For bare iron asteroids (Fig. 2) meteoroid drag is less effective, though it could still exceed the Yarkovsky effect at small target sizes if $60 \mathrm{~km} / \mathrm{s}$ impacts take place in the brittle rather than ductile deformation regimes (that is, if $\alpha$ is larger than assumed here). In Fig. 2, we account for the increased density of the target $\left(\rho_{a}=8000 \mathrm{~kg} \mathrm{~m}^{-3}\right.$ instead of 3500) but assume $\alpha=10$.

For regolith covered rock (Fig. 3), the drag effect considered here falls far below the diurnal Yarkovsky effect, near the seasonal. The lower thermal conductivity of the regolith-covered body enhances the Yarkovsky effect by increasing the day-night temperature difference.

Since small asteroids tend to be more quickly rotating and have smaller 
gravitational attractions, it has been argued that they are unlikely to have substantial regolith (Farinella et al., 1998). If this is correct and meter-class stony asteroids do not usually have regolith coatings, then meteoroid impact drag will compete with Yarkovsky effect in importance for stony meteorite delivery to Earth. It will be less important in the delivery of regolith-covered bodies or iron asteroids.

\subsection{Uncertainties}

Many unknowns also cloud the true importance of the effect of meteoroid impact drag. Microcratering experiments seldom reach impact speeds beyond 10 $\mathrm{km} / \mathrm{s}$ due to the difficulty in accelerating the projectile. The meteoroid impacts considered here are much faster: does this increase or decrease the effective drag force? The meteoroid environment at the asteroid belt is uncertain, and our estimate could easily be off by an order of magnitude. Even the meteoroid flux at Earth is not particularly well known. An earlier measurement of the flux of impactors by (Grün et al., 1985) had a number three times smaller than that of Love \& Brownlee (1993). In the opposite direction, the slope of the differential mass distribution of meteoroids near the Earth is close to -2 (Blaauw et al., 2011; Campbell-Brown \& Braid, 2011). Values less than -2 imply most of the mass is in the smallest particles (which is what we've assumed here) while a value of precisely -2 means that each decade of mass contributes equally. The proximity of the slope to -2 means that our choice of typical impactor mass may be an underestimate and may skew our projections downwards.

The physics of microcratering on an asteroidal target provide other unknowns in terms of shape, composition and strength. For example, impacts typically eject material on average perpendicular to the normal of the surface except for the most oblique impacts (Vedder, 1971). This effect will lessen the back-reaction when the impact takes place on the limb of the target. On the other hand, hypervelocity impactors are known to produce secondary craters (Hörz et al., 1971), which may themselves release more mass, particularly if the impact takes place into a pre-existing concavity such as a crater. The breakage 


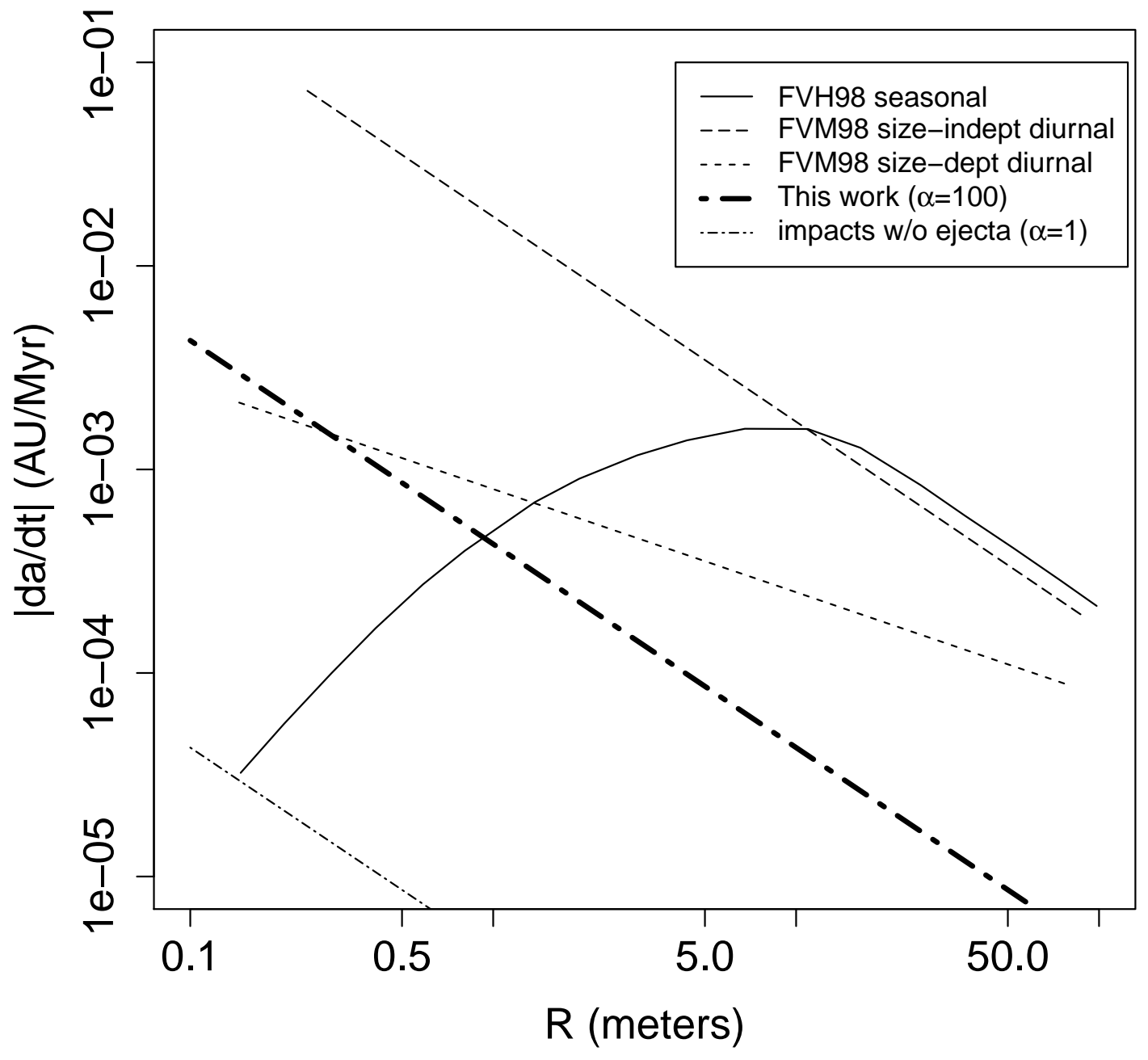

Figure 1: The maximum semimajor axis drift for bare basalt fragment at $2 \mathrm{AU}$. The curves for the seasonal and diurnal Yarkovsky effect, either with a size-independent spin period of $5 \mathrm{~h}$ or with spin rate proportional to $1 / R$ are adapted from Farinella et al. (1998) Figure 1. 


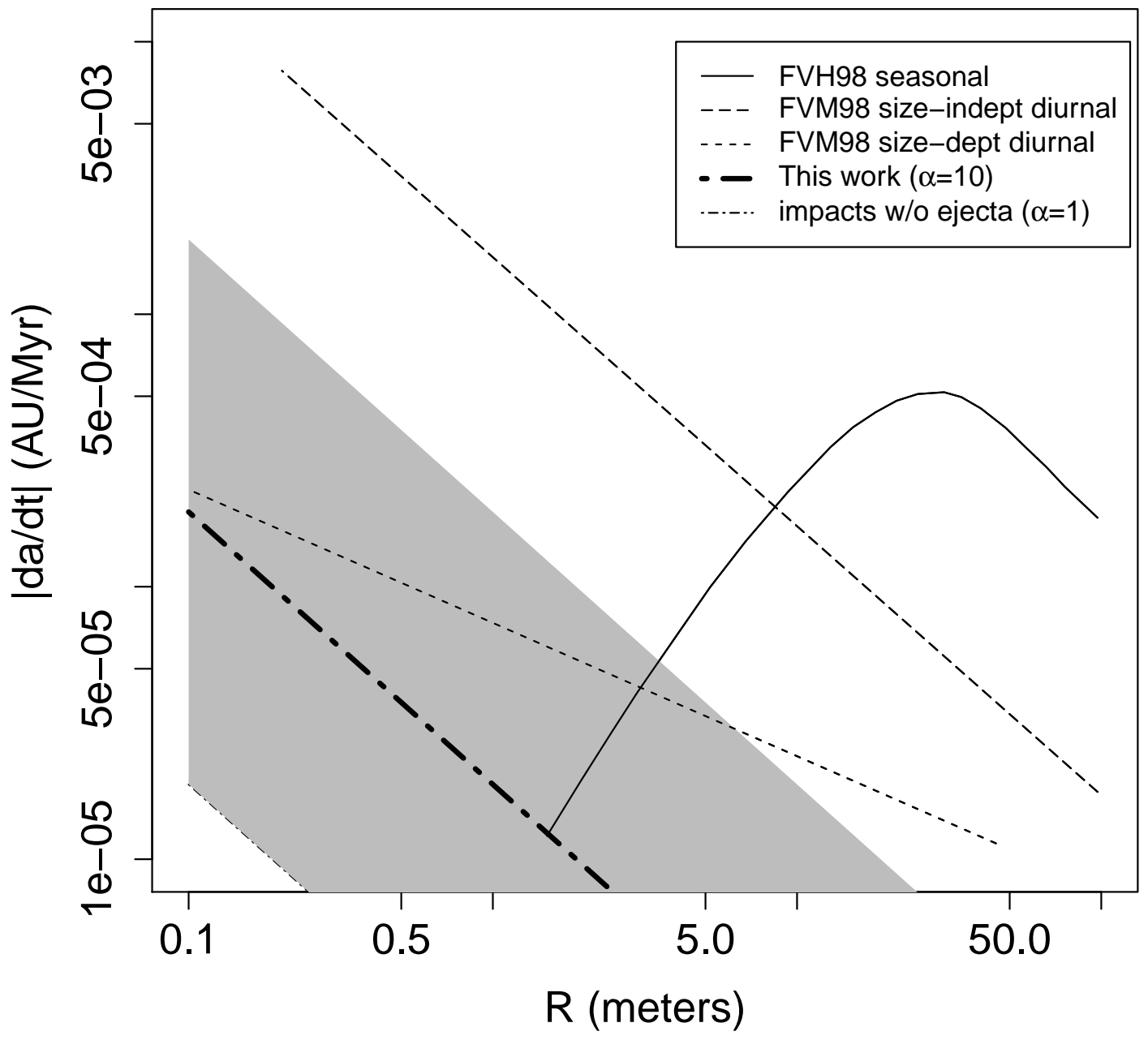

Figure 2: The maximum semimajor axis drift for bare iron fragment at $2 \mathrm{AU}$. The curves for the seasonal and diurnal Yarkovsky effect, either with a size-independent spin period of $5 \mathrm{~h}$ or with spin rate proportional to $1 / R$ are adapted from Farinella et al. (1998) Figure 3. A grey region indicates a factor of 10 around the meteoroid drag line, indicating the high level of uncertainty in this value. 


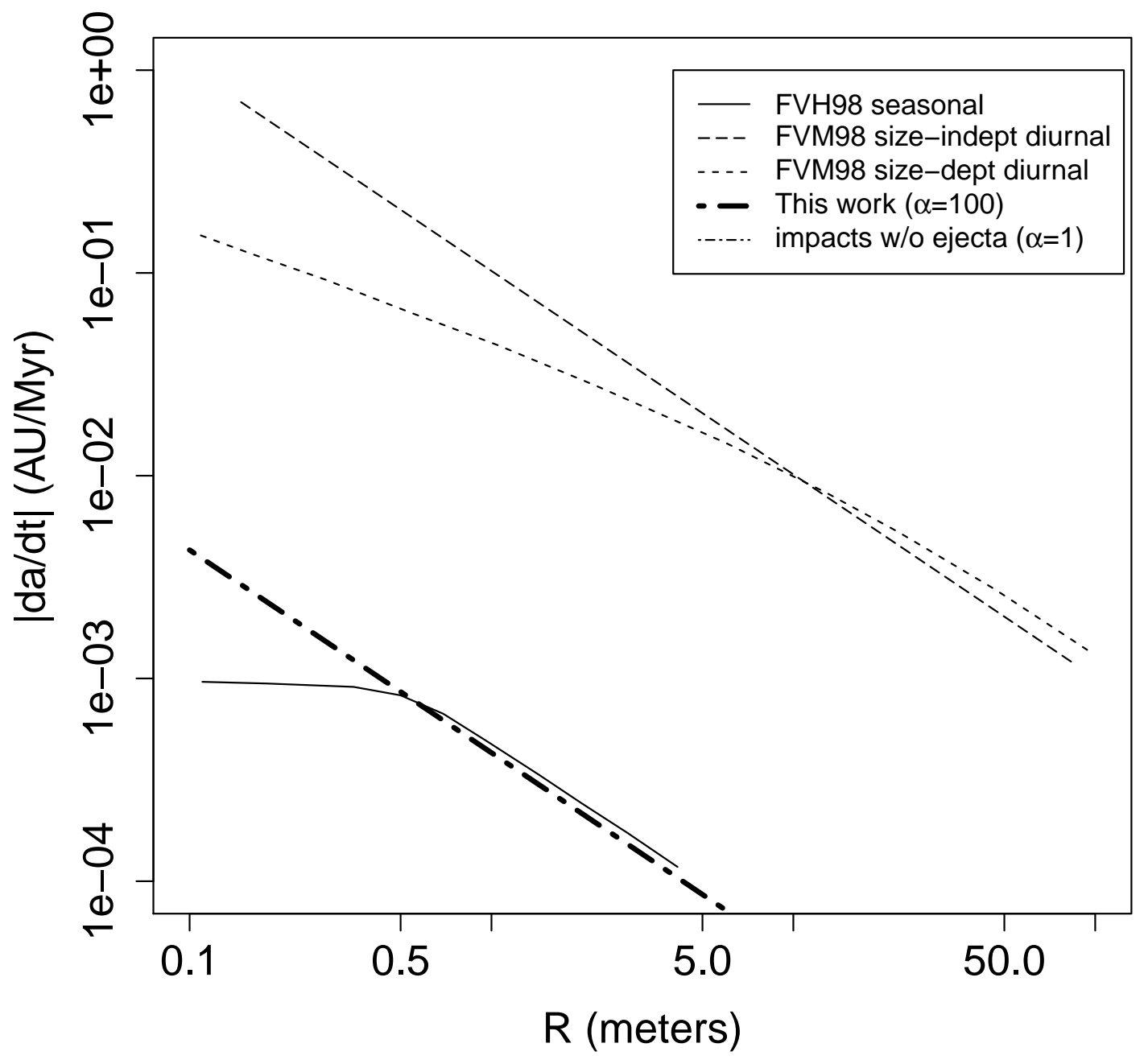

Figure 3: The maximum semimajor axis drift for a regolith-covered fragment at 2 AU. The curves for the seasonal and diurnal Yarkovsky effect, either with a size-independent spin period of $5 \mathrm{~h}$ or with spin rate proportional to $1 / R$ are adapted from Farinella et al. (1998) Figure 4. 
of edges and the release of unconsolidated material, the rupture of the body or pieces thereof (Gault et al., 1972) may serve to increase the total momentum released by an impact.

\subsection{Rotational state of the parent}

The two varieties of the Yarkovsky effect, the traditional or 'diurnal' (Öpik, 1951; Peterson, 1976) and 'seasonal' (Rubincam, 1995, 1998) affect asteroid orbits differently. The diurnal effect can either increase or decrease $a$ depending on the body's rotation state. The seasonal effect always acts to decrease the semimajor axis, though its magnitude also depends on the orientation of the asteroid's rotation pole relative to the Sun. The impact drag effect considered here is independent of the rotation state of the asteroid. However meteoroid impacts can re-orient the spin of the asteroid and thus may play an important indirect role in the Yarkovsky effect itself.

The order-of-magnitude assumption that is usually made in calculating the time between pole reorientations involves equating the rotational angular momentum of the asteroid to that imparted by the impactor to determine the minimum size needed (at some typical encounter velocity) to perform such a reorientation. A knowledge of the size distribution of the impactors then allows the frequency of such impacts to be estimated. However, the momentum carried by the ejecta also comes into play here: the rotational momentum imparted by the impactor is not just its own momentum times the radius of the target, but $\alpha$ times as much. Though many poorly-understood effects (e.g. asteroid composition, shape, internal cohesiveness, the direction of debris ejection under impacts near the limb of the asteroid, etc. ) would come into play in a detailed calculation, to first order the angular momentum transferred to the asteroid is increased by a factor of $\alpha$ over that usually assumed, which means that a particle of only $\alpha^{-1}$ of the mass or $\alpha^{-1 / 3}$ the radius can effect the same rotational change. For $\alpha \sim 100$, this translates into a decrease by a factor of $100^{1 / 3} \approx 4.6$ in radius. Given that the cumulative distribution of impactor sizes goes something like $R^{-5 / 2}$ (Dohnanvi, 1969) this translates to reorientation 
events occurring $\alpha^{5 / 6}=100^{5 / 6} \approx 46$ times more frequently.

Thus, the net effectiveness of the diurnal variant of the Yarkovsky effect in particular may be considerably reduced. Meteoroid impacts do not reduce the height of the Yarkovsky curves in Fig. 1]- 3, instead they cause the sign of the effect to change more frequently. If the distance between the asteroid and the resonance is $\Delta a$, then the escape process will be of the nature of a random walk if the time between pole reorientations $\tau_{r o t}$ times $\dot{a}$ is greater than $\Delta a$. The more frequently reorientations occur, the more asteroids will be in the randomwalk regime. Such asteroids will have a net Yarkovsky drift that proceeds at a rate that is diminished by roughly one over square root of $\alpha^{5 / 6}(\sim 0.15 \sim 1 / 7)$. This additional factor may mean the smaller but consistently directed effect of meteoroid impacts can compete with the diurnal Yarkovsky effect at even larger sizes than Fig. 1]- 3 would imply.

\section{The YORP effect}

The Yarkovsky-O'Keefe-Radzievskii-Paddack (YORP) effect can change an asteroid's spin through the uneven re-radiation of thermal photons. Meteoroid impacts onto an asteroidal surface can change the rotation rate of the target at rates which are below (but perhaps uncomfortably close to) those currently being reported for YORP detections in near-Earth asteroids.

The angular momentum transferred to the target in a single impact is roughly $\Delta L=\alpha m v R$, while the target's initial angular momentum is $L=I \omega$ where $I$ is its moment of inertia (here taken to be that of a sphere $I=\frac{2}{5} M R^{2}$ ) and $\omega$ is its angular rate of rotation, related to its period $P$ through $P=2 \pi / \omega$. The fractional change in angular momentum from a single impact is

$$
\begin{aligned}
\frac{\Delta L}{L} & =\frac{\alpha m v R}{\omega\left(\frac{2}{5} M R^{2}\right)} \\
& =\frac{15 \alpha m v P}{16 \pi^{2} \rho_{a} R^{4}}
\end{aligned}
$$

If we ignore the small change in the moment of inertia of the target under the erosive effect of the impacts, then change in angular momentum produces a 
concomitant change in the rotation rate of the target, $\Delta \omega / \omega \approx \Delta L / L$. However, since individual impacts occur randomly on the surface, they are as likely to speed up the rotation as slow it down.

Let's consider the first reported detection of the YORP effect by Lowry et al. (2007); Taylor et al. (2007). The asteroid (54509) 2000 PH5 was observed to have its period decreasing at a fractional rate of $-1.7 \times 10^{-6}$ per year. Assuming a mean radius of $57 \mathrm{~m}$ and a rotation period of 730 seconds for 2000 PH5, Eq. 16 becomes

$\frac{\Delta \omega}{\omega} \approx 1.6 \times 10^{-10}\left(\frac{\alpha}{100}\right)\left(\frac{\rho_{a}}{3500 \mathrm{~kg} \mathrm{~m}^{3}}\right)^{-1}\left(\frac{m}{1.5 \times 10^{-8} \mathrm{~kg}}\right)\left(\frac{v}{\left.60 \mathrm{~km} \mathrm{~s}^{(1-1} 7\right)}\right.$

so each impact affects the rotation rate by only one part in $10^{10}$. Though minuscule, this is still much larger than the ratio of the impactor to target masses, which is of order $10^{-17}$, and hints that meteoroids may be more effective at changing asteroid rotation rates than might initially be assumed. The difference arises from the high speed of the impactor relative to the rotation of the target: material even on the surface of 2000 PH5 moves no quicker than about $0.5 \mathrm{~m} / \mathrm{s}$ under rotation. The impactor contains $10^{5}$ times as much momentum per unit mass as the target material, and momentum transport by ejecta multiplies it further.

The asteroid suffers an impact rate $s n \pi R^{2}$ which in this case results in approximately 5000 impacts per year on 2000 PH5. Assuming a simple onedimensional random walk, the net fractional change in the period of order $10^{-8}$ per year. This is two orders of magnitude below the fractional change in period observed $\left(-1.7 \times 10^{-6} \mathrm{yr}^{-1}\right)$ and so small apex meteoroids do not affect the spin rate at the same level as YORP in this case.

A more recent determination of the YORP effect on asteroid (25143) Itokawa (Lowry et al., 2014) is also essentially unaffected by impacts from apex meteoroids. The rotation period is 12.14 hours and its mean radius, $162 \mathrm{~m}$ (Scheeres et al., 2007). Despite its larger size, because of its slower rotation, the fractional change in angular momentum per impact is also $1.5 \times 10^{-11}$. Itokawa's larger size means that the rate of impacts is slightly higher, approximately $4 \times 10^{4}$ per year, or a 
fractional change under a random walk of $3 \times 10^{-8} \mathrm{yr}^{-1}$. This is equivalent to a change in the rotation period of $\sim 1$ millisecond over the course of one year, much less than the value reported by Lowry et al. (2014) of $\sim 45 \mathrm{~ms} \mathrm{yr}^{-1}$.

The $R^{-4}$ dependence of Eq. 16 implies that the rotation states of smaller asteroids are more susceptible to change by meteoroid impacts. If the random walk goes like the square root of number of impacts $\left(\propto R^{2}\right)$, then the net effect should go like $R^{-3}$. This means that for the calculated effect of meteoroids of $1 \mathrm{~ms} \mathrm{yr}^{-1}$ to increase to $45 \mathrm{~ms} \mathrm{yr}^{-1}$, a decrease in the asteroid size by only a factor of four is required. Thus the impact of high-speed apex meteoroids may significantly influence the rotation state (at least to the same degree as YORP) for asteroids smaller than a few tens of meters in size.

\subsection{Single impacts}

The relatively large effect that a single meteoroid can have raises the question of the smallest meteoroid impact that would produce a result comparable to that of YORP. Here we will examine the single impact required to create a fractional change in period of one part in $10^{6}$ in 2000 PH5, comparable to that reported by Lowry et al. (2007) and Tavlor et al. (2007) for the YORP effect.

Rearranging Eq. 16 gives a minimum impactor mass $m$ needed to produce a given fractional change in rotation rate

$$
\begin{aligned}
m= & \left(\frac{\Delta \omega}{\omega}\right) \frac{16 \pi^{2} \rho_{a} R^{4}}{15 \alpha v P} \\
& 8.9 \times 10^{-5} \mathrm{~kg}\left(\frac{\Delta \omega / \omega}{10^{-6}}\right)\left(\frac{\alpha}{100}\right)^{-1}\left(\frac{\rho_{\mathrm{a}}}{3500 \mathrm{~kg} \mathrm{~m}^{-3}}\right)\left(\frac{\mathrm{v}}{60 \mathrm{~km} / \mathrm{s}}\right)^{-1}
\end{aligned}
$$

At $60 \mathrm{~km} / \mathrm{s}$ impact speed, a fractional change in period of $10^{-6}$ could be generated by a single a $0.09 \mathrm{~g}$ meteoroid if $\alpha=100$. Such a particle is approximately $2 \mathrm{~mm}$ in radius at a density of $2500 \mathrm{~kg} \mathrm{~m}^{-3}$.

The apex sources are rich in small particles but not in large ones. Though $2 \mathrm{~mm}$ radius particles certainly occur there, it is clear that in considering the relatively large asteroids examined here, impacts by larger particles will be more effective. We instead consider the effect of the sporadic meteoroid population as a whole, which has somewhat lower speeds but more larger particles. 
The encounter velocities between typical sporadic meteoroids and the Earth are lower $(\sim 30 \mathrm{~km} / \mathrm{s}$, Campbell-Brown (2008)) than for apex meteoroids and so the impact speeds for asteroids on near-circular prograde orbits near our planet are reduced as well. The excavated mass rises in Eq. 14 due to the larger projectile mass but decreases by a comparable amount due to the smaller impact speed, so we continue to adopt $\alpha=100$. At this reduced speed, a mass of 0.18 $\mathrm{g}$ (Eq. 19) is required to affect a spin change of one part in $10^{6}$ in $2000 \mathrm{PH} 5$.

Using video recordings of meteors in Earth's atmosphere, Campbell-Brown \& Braid (2011) found the total sporadic meteor flux to be $0.18 \pm 0.04 \mathrm{~km}^{-2} \mathrm{hr}^{-1}(5 \times$ $10^{-11} \mathrm{~m}^{-2} \mathrm{~s}^{-1}$ ) down to a limiting mass of $2 \times 10^{-6} \mathrm{~kg}$ and deduced a differential mass slope of $-2.02 \pm 0.02$. The cumulative size distribution is then proportional to the impactor size to the -3.06 , which is steeper than the standard Dohnanyi -2.5 value: using the Dohnanyi value would overestimate the impact rate somewhat.

A differential mass slope of near -2 implies that the cumulative impact rate is inversely proportional to the mass, and so the rates of impacts by meteoroids of at least $1.8 \times 10^{-4} \mathrm{~kg}$ is approximately $\left(1.8 \times 10^{-4} / 2 \times 10^{-6}\right)^{-1}\left(5 \times 10^{-11}\right)=$ $5 \times 10^{-13} \mathrm{~m}^{-2} \mathrm{hr}^{-1}$, or one every few years on $2000 \mathrm{PH} 5$, which can be neglected.

The change in period reported for Itokawa (Lowry et al., 2014) is similar, $45 \mathrm{~ms}$ in 12.14 hours or $\Delta P / P \sim 10^{-6}$. Though it is a larger body, owing to its slower rotation rate, an impactor of about the same mass as for the 2000 PH5 case is needed. Itokawa has a larger cross section though, and might see one such impact per year (assuming a mean radius of $162 \mathrm{~m}$ ), a rate which is arguably not entirely negligible.

Though meteoroid impacts may have an important role to play, we are not asserting here that the spin changes attributed to YORP have actually been produced by meteoroid impacts, for two reasons. First, the observed changes in rotation are seen to be accelerating, which is more compatible with YORP than impacts (though admittedly a small number of impacts could conspire to look like a net acceleration in the short term). Secondly, the analysis above uses a value of $\alpha$ of 100 deduced for bare rock. Itokawa at least is certainly regolith- 
covered (e.g. Fujiwara et al., 2006) which is likely to reduce $\alpha$. If the impact rate is goes like the inverse of the impactor mass, then the rate of impacts capable of generating the required change in period goes roughly like $\alpha$. A small reduction in $\alpha$ would then reduce the rate of impacts of concern to one every several years, which can be neglected. Nonetheless, we do conclude that the effect of impacts is at a level which demands some attention when sensitive measurements of asteroid spins are being made.

\section{Other considerations}

\subsection{Other sporadic meteor sources and radiation pressure}

There are six generally accepted sporadic meteoroid sources, that is, six broad inhomogeneities in the time-averaged meteoroid environment seen by the moving Earth (e.g. Stohl, 1986; Brown \& Jones, 1995; Chau et al., 2007; Campbell-Brown, 2008). The north and south apex sources have been the basis of the analysis so far. Two others are the north and south toroidal sources, which also arrive at the Earth from the direction roughly opposite its motion but at higher ecliptic latitudes. These may contribute to the drag force considered here but are generally weaker than the apex sources and will be neglected here. The two remaining sources are the helion and antihelion sources, consisting of particles on high-eccentricity orbits which hit the Earth from the directions of the Sun and of opposition respectively. These particles may create a small radial force component on asteroids but have been ignored here so far because the helion and antihelion sources have roughly equal strengths, and so the net force from them will average out. Though it is perhaps worth noting that the strengths are not precisely equal, the antihelion source may in fact be stronger but probably only by about 20-30\% (see Wiegert et al. (2009) and references therein.)

One might wonder if the helion or antihelion meteoroid sources, owing to the radial nature of the forces they create, could confound measurements of the radiation pressure on small asteroids, which have been used to determine 
their densities in particular cases (Micheli et al., 2012, 2013, 2014). We can show that the meteoroid drag as considered here is much smaller than radiation pressure near the Earth. Even if we ignore the opposing nature of the helion and antihelion sources and assume that one or the other constitutes all of the meteoroid impacts considered in Eq. 1, the effect is less than radiation pressure. Adopting the expression of Burns et al. (1979) for the ratio of radiation pressure to solar gravity and combining that with Eq. 1 yields a ratio of radiation to meteoroid-derived accelerations $\beta_{m}$ of

$$
\beta_{m}=\frac{L_{\odot} Q_{P R}}{4 \pi c s n m v r^{2} \alpha} \sim 7 \times 10^{3}
$$

where $L_{\odot}$ is the solar luminosity, $Q_{P R}$ is a radiation absorption coefficient we have taken to be unity, $c$ is the speed of light, $m$ and $v$ are the impactor mass and velocities and $r$ is the heliocentric distance. Here we have adopted $s=0.5$ and an impact velocity $v=30 \mathrm{~km} / \mathrm{s}$ more appropriate for the helion and antihelion sources but this makes little difference; the effect of meteoroid drag is much smaller than radiation pressure under all reasonable conditions.

\subsection{Erosion rates}

The large amounts of ejecta produced by meteoroid impacts also contribute to the erosion of the target body. The $1.5 \times 10^{-8} \mathrm{~kg}$ impactor considered here releases $3.1 \times 10^{-4} \mathrm{~kg}$ of ejecta if $N=2 \times 10^{4}$. At the Earth's orbit this translates into a rough survival time $\tau$ against erosion of a stony body

$$
\tau \sim 16 \mathrm{Myr}\left(\frac{\rho_{a}}{3500 \mathrm{~kg} \mathrm{~m}^{-3}}\right)\left(\frac{R}{1 \mathrm{~m}}\right)
$$

where this simple expression is an upper limit, as it ignores the decreasing impact rate as the target size decreases. This time is comparable to the dynamical lifetimes (10 Myr, Gladman et al. (1997, 2000) ) of near-Earth asteroids and so the high levels of ejecta production assumed here do not conflict with reality on this basis. The high erosion rates proposed here are also consistent with cosmic ray exposure ages of meteorites. The cosmic ray exposure ages of stony meteorites rarely exceed 100 Myr (Herzog, 2005) which can be accommodated in Eq. 21 by a parent body of ten meters in size. 


\section{Conclusions}

We have discussed the effect of the meteoroid environment on small asteroids. It was argued that the net effect of such impacts is enhanced by up to two orders of magnitude by the momentum transported by ejecta. Careful examination of the physics of hypervelocity impacts will be needed to determine the exact magnitude of the effect and its broader role in asteroid evolution.

The instantaneous value of the net drag produced by the apex meteoroids is found to be typically smaller than that of the variants of Yarkovsky effect except at sizes well below one meter. The effect is independent of thermal or rotational properties of individual asteroids, though not their densities. Independence from the rotation state means that the meteoroid environment acts consistently as a drag and cannot increase an asteroid's semimajor axis. Impacts also serve to reorient the spin axis of the target and thus can decrease the net effectiveness of Yarkovsky drift.

The meteoroid impacts have the potential to confuse measurements of the YORP effect. Here the effect is primarily due to larger (centimeter) sized particles from the general sporadic meteoroid population. However we conclude that impacts have probably not clouded recent measurements of YORP among the near-Earth asteroid population, though it will be an important consideration when measurements of smaller (10 meter class) bodies are made. In fact, high precision asteroid spin measurements may be sensitive enough to measure the effect of meteoroid impacts on spin states.

The effects of meteoroid impacts on the dynamics of small asteroids remains to be worked out in detail. If the momentum transport by ejecta proposed here correctly represents real meteoroid-asteroid collisions, then the meteoroid impacts may prove to be as important as radiative effects in the dynamical evolution of small asteroids and the transport of meteorites to Earth. 


\section{Acknowledgements}

This work was performed in part with funding from the Natural Sciences and Engineering Research Council of Canada.

\section{References}

\section{References}

Blaauw, R. C., Campbell-Brown, M. D., \& Weryk, R. J. (2011). Mass distribution indices of sporadic meteors using radar data. MNRAS, 412, 2033-2039. doi $10.1111 / \mathrm{j} .1365-2966.2010 .18038 . \mathrm{x}$

Braslau, D. (1970). Partitioning of energy in hypervelocity impact against loose sand targets. J. Geophys. Res., 75, 3987-3999. doi:10.1029/JB075i020p03987

Brown, P., \& Jones, J. (1995). A Determination of the Strengths of the Sporadic Radio-Meteor Sources. Earth Moon and Planets, 68, 223-245. doi:10.1007/BF00671512

Burns, J. A., Lamy, P. L., \& Soter, S. (1979). Radiation forces on small particles in the solar system. Icarus, 40,1-48. doi:10.1016/0019-1035(79)90050-2.

Campbell-Brown, M. D. (2008). High resolution radiant distribution and orbits of sporadic radar meteoroids. Icarus, 196, 144-163. doi $10.1016 / j$. icarus.2008.02.022

Campbell-Brown, M. D., \& Braid, D. (2011). Video Meteor Fluxes. In W. J. Cooke, D. E. Moser, B. F. Hardin, \& D. Janches (Eds.), Meteoroids: The Smallest Solar System Bodies (pp. 304-312).

Chau, J. L., \& Woodman, R. F. (2004). Observations of meteor head-echoes using the jicamaraca $50 \mathrm{mhz}$ radar in interferometer mode. Atmos. Chem. Phys, 4, 511-521. 
Chau, J. L., Woodman, R. F., \& Galindo, F. (2007). Sporadic meteor sources as observed by the Jicamarca high-power large-aperture VHF radar. Icarus, 188, 162-174. doi:10.1016/j.icarus.2006.11.006.

Comerford, M. F. (1967). Comparative erosion rates of stone and iron meteorites under small-particle bombardment. Geochimica et Cosmochimica Acta, 31, $1457-1471$.

Dohnanyi, J. S. (1969). Collisional model of asteroids and their debris. $J$. Geophys. Res., 74, 2531-2554.

Farinella, P., Vokrouhlicky, D., \& Hartmann, W. K. (1998). Meteorite Delivery via Yarkovsky Orbital Drift. Icarus, 132, 378-387. doi:10.1006/icar.1997.5872.

Fechtig, H., Gault, D. E., Neukum, G., \& Schneider, E. (1972). Laboratory simulation of lunar craters. Naturwissenschaften, 59, 151-157. doi $10.1007 / \mathrm{BF} 00637353$

Fujiwara, A., Kawaguchi, J., Yeomans, D. K., Abe, M., Mukai, T., Okada, T., Saito, J., Yano, H., Yoshikawa, M., Scheeres, D. J., Barnouin-Jha, O., Cheng, A. F., Demura, H., Gaskell, R. W., Hirata, N., Ikeda, H., Kominato, T., Miyamoto, H., Nakamura, A. M., Nakamura, R., Sasaki, S., \& Uesugi, K. (2006). The Rubble-Pile Asteroid Itokawa as Observed by Hayabusa. Science, 312, 1330-1334. doi 10.1126/science.1125841.

Galligan, D. P., \& Baggaley, W. J. (2004). The orbital distribution of radardetected meteoroids of the Solar system dust cloud. MNRAS, 353, 422-446. doi $10.1111 / j .1365-2966.2004 .08078 . x$

Gault, D. E. (1973). Displaced mass, depth, diameter, and effects of oblique trajectories for impact craters formed in dense crystalline rocks. Moon, 6, 32-44. doi $10.1007 / \mathrm{BF} 02630651$.

Gault, D. E., Hörz, F., \& Hartung, J. B. (1972). Effects of microcratering on the lunar surface. In A. E. Metzger, J. I. Trombka, L. E. Peterson, R. C. Reedy, 
\& J. R. Arnold (Eds.), Lunar and Planetary Science Conference Proceedings (p. 2713). volume 3 of Lunar and Planetary Science Conference Proceedings.

Gladman, B., Michel, P., \& Froeschlé, C. (2000). The Near-Earth Object Population. Icarus, 146, 176-189. doi:10.1006/icar.2000.6391.

Gladman, B. J., Migliorini, F., Morbidelli, A., Zappala, V., Michel, P., Cellino, A., Froeschle, C., Levison, H. F., Bailey, M., \& Duncan, M. (1997). Dynamical lifetimes of objects injected into asteroid belt resonances. Science, 277, 197201.

Grün, E., Zook, H. A., Fechtig, H., \& Giese, R. H. (1985). Collisional balance of the meteoritic complex. Icarus, 62, 244-272. doi:10.1016/0019-1035(85)90121-6.

Hartmann, W. K. (1983). Energy Partitioning in Impacts Into Regolith-Like Powders. In Lunar and Planetary Science Conference (pp. 281-282). volume 14 of Lunar and Planetary Science Conference.

Hartmann, W. K. (1985). Impact experiments. I - Ejecta velocity distributions and related results from regolith targets. Icarus, 63, 69-98. doi $10.1016 / 0019-1035(85) 90021-1$.

Herzog, G. F. (2005). Cosmic-ray Exposure Ages of Meteorites. In A. M. Davis, H. D. Holland, \& K. K. Turekian (Eds.), Meteorites, Comets and Planets: Treatise on Geochemistry, Volume 1. Edited by A. M. Davis. Executive Editors: H. D. Holland and K. K. Turekian. ISBN 0-08-044720-1. Published by Elsevier B. V., Amsterdam, The Netherlands, 2005, p.347 (p. 347). Elsevier B.

Hörz, F., Hartung, J. B., \& Gault, D. E. (1971). Micrometeorite craters on lunar rock surfaces. J. Geophys. Res., 76, 5770-5798. doi $10.1029 /$ JB076i023p05770

Hunt, S. M., Oppenheim, M., Close, S., Brown, P. G., McKeen, F., \& Minardi, M. (2004). Determination of the meteoroid velocity dis- 
tribution at the Earth using high-gain radar. Icarus, 168, 34-42. doi $10.1016 /$ j.icarus.2003.08.006

Janches, D., Nolan, M. C., Meisel, D. D., Mathews, J. D., Zhou, Q. H., \& Moser, D. E. (2003). On the geocentric micrometeor velocity distribution. Journal of Geophysical Research (Space Physics), 108, 1222-1235. doi $10.1029 / 2002$ JA009789.

Jones, J., \& Brown, P. (1993). Sporadic meteor radiant distributions - Orbital survey results. MNRAS, 265, 524-532.

Love, S. G., \& Brownlee, D. E. (1993). A Direct Measurement of the Terrestrial Mass Accretion Rate of Cosmic Dust. Science, 262, 550.

Lowry, S. C., Fitzsimmons, A., Pravec, P., Vokrouhlický, D., Boehnhardt, H., Taylor, P. A., Margot, J.-L., Galád, A., Irwin, M., Irwin, J., \& Kusnirák, P. (2007). Direct Detection of the Asteroidal YORP Effect. Science, 316, 272-. doi $10.1126 /$ science. 1139040

Lowry, S. C., Weissman, P. R., Duddy, S. R., Rozitis, B., Fitzsimmons, A., Green, S. F., Hicks, M. D., Snodgrass, C., Wolters, S. D., Chesley, S. R., Pittichová, J., \& van Oers, P. (2014). The internal structure of asteroid (25143) Itokawa as revealed by detection of YORP spin-up. A\&3A, 562, A48. doi $10.1051 / 0004-6361 / 201322602$.

Micheli, M., Tholen, D. J., \& Elliott, G. T. (2012). Detection of radiation pressure acting on 2009 BD. New Astronomy, 17, 446-452. doi $10.1016 /$ j.newast.2011.11.008 arXiv:1106.0564

Micheli, M., Tholen, D. J., \& Elliott, G. T. (2013). 2012 LA, an optimal astrometric target for radiation pressure detection. Icarus, 226, 251-255. doi $10.1016 / j$. icarus.2013.05.032

Micheli, M., Tholen, D. J., \& Elliott, G. T. (2014). Radiation Pressure Detection and Density Estimate for 2011 MD. Astrophys. J. Lett., 788, L1. doi $10.1088 / 2041-8205 / 788 / 1 /$ L1. arXiv: 1403.6033 
Öpik, E. (1951). Collision probability with the planets and the distribution of planetary matter. Proc. R. Irish Acad. Sect. A, vol. 54, p. 165-199 (1951)., 54, 165-199.

Peterson, C. (1976). A source mechanism for meteorites controlled by the Yarkovsky effect. Icarus, 29, 91-111. doi 10.1016/0019-1035(76)90105-6.

Rabinowitz, D. L. (1993). The size distribution of earth-approaching asteroids. ApJ , 407, 412-427.

Rabinowitz, D. L., Gehrels, T., Scotti, J. V., McMillan, R. S., Perry, M. L., Wisniewski, W., Larson, S. M., Howell, E. S., \& Mueller, B. E. A. (1993). Evidence for a near-Earth asteroid belt. Nature, 363, 704-706. doi $10.1038 / 363704 \mathrm{a} 0$

Roy, A. E. (1978). Orbital Motion. Bristol: Adam Hilger Ltd.

Rubincam, D. P. (1995). Asteroid orbit evolution due to thermal drag. $J$. Geophys. Res., 100, 1585-1594. doi:10.1029/94JE02411.

Rubincam, D. P. (1998). Yarkovsky thermal drag on small asteroids and MarsEarth delivery. J. Geophys. Res., 103, 1725-1732. doi 10.1029/97JE03034

Sato, T., Nakamura, T., \& Nishimura, K. (2000). Orbit determination of meteors using the MU radar. IEICE Trans. Commun., E83-B(9), 1990-1995.

Schaeffer, O. A., Nagel, K., Neukum, G., \& Fechtig, H. (1979). Effects of Micrometeorite Bombardment on Cosmic Ray Ages of Stony and Iron Meteorites: Evidence for a Long Term Temporal Change in Cosmic Ray Intensity. In Lunar and Planetary Science Conference (pp. 1061-1062). volume 10 of Lunar and Planetary Science Conference.

Scheeres, D. J., Abe, M., Yoshikawa, M., Nakamura, R., Gaskell, R. W., \& Abell, P. A. (2007). The effect of YORP on Itokawa. Icarus, 188, 425-429. doi $10.1016 / \mathrm{j}$. icarus.2006.12.014 
Stohl, J. (1986). Seasonal Variation in the Radiant Distribution of Meteors. In L. Kresak, \& P. M. Millman (Eds.), Physics and Dynamics of Meteors (pp. 298-303). volume 33 of IAU Symposium.

Taylor, A. D. (1995). The Harvard Radio Meteor Project velocity distribution reappraised. Icarus, 116, 154-158. doi:10.1006/icar.1995.1117.

Taylor, P. A., Margot, J.-L., Vokrouhlický, D., Scheeres, D. J., Pravec, P., Lowry, S. C., Fitzsimmons, A., Nolan, M. C., Ostro, S. J., Benner, L. A. M., Giorgini, J. D., \& Magri, C. (2007). Spin Rate of Asteroid (54509) 2000 PH5 Increasing Due to the YORP Effect. Science, 316, 274-. doi $10.1126 /$ science. 1139038

Vedder, J. F. (1971). Microcraters in glass and minerals. Earth and Planetary Science Letters, 11, 291-296. doi 10.1016/0012-821X(71)90182-8.

Vedder, J. F. (1972). Craters formed in mineral dust by hypervelocity microparticles. J. Geophys. Res., 77, 4304-4309. doi 10.1029/JB077i023p04304.

Weidenschilling, S. J., \& Jackson, A. A. (1993). Orbital resonances and Poynting-Robertson drag. Icarus, 104, 244-254.

Wiegert, P., Vaubaillon, J., \& Campbell-Brown, M. (2009). A dynamical model of the sporadic meteoroid complex. Icarus, 201, 295-310. doi $10.1016 / \mathrm{j}$. icarus.2008.12.030 